\title{
P03-6-18 Poster session
}

\section{Programmed Necrosis of Adipocytes Induced by High-dose Osteocalcin}

\author{
Masato Hirata ${ }^{1}$, Takahito Otani ${ }^{1}$, Akiko Mizokami ${ }^{2}$, Norio Kitagawa ${ }^{1}$, Eijiro Jimi ${ }^{2}$, Tetsuichiro Inai ${ }^{1}$
}

${ }^{I}$ Fukuoka Dental College, Japan, ${ }^{2}$ Kyushu University, Japan

Osteocalcin, one of non-collagenous bone matrix proteins, is known to regulate the energy metabolism in the uncarboxylated glutamate form designated as GluOC. We examined the effects of GluOC using 3T3-L1 adipocytes, and revealed the signaling pathway by which GluOC ranging $1 \mathrm{ng} / \mathrm{ml}$ to $10 \mathrm{ng} / \mathrm{ml}$ increased the expressions of adiponectin, an adipokine regulating the metabolism of glucose and lipids, and peroxisome proliferator-activated receptor-gamma (PPARgamma), a master regulator of adipogenesis, via its putative receptor GPRC6A. By contrast, we also found that the expression of adiponectin was inhibited when GluOC at the concentration over $20 \mathrm{ng} / \mathrm{ml}$ was used. In the present study, we explored the mechanisms responsible for the inhibition; Adipocytes showed typical morphology of programmed necrosis (necroptosis) as assessed by microscopic and immunohistochemical observations. The adipocytes bound by GluOC via GPRC6A increased the expression of FoxO1, a transcription factor regulating cell death, via cAMP-PKA-ERKCREB signaling axis along with activation of $\mathrm{p} 300$, a transcriptional coactivator of CREB, followed by the expression of FasL, a member of tumor necrosis factor superfamily. FasL expressed in the plasma membrane triggered Fas activation followed by the phosphorylations and trimerization of mixed lineage kinase domain-like protein (MLKL), a major mediator of necroptosis. Consequently, calcium influx and reactive oxygen species (ROS) production were triggered in the downstream of MLKL activation leading to necroptosis. Although high-dose of GluOC decreased the cell number of adipocytes, it was maximally about by $33 \%$ even after $96 \mathrm{~h}$, probably because cell-cell physical contact was required for necroptosis via Fas/FasL. We have here revealed the signaling pathway by which high-dose of GluOC induced necroptosis in 3T3-L1 adipocytes. 